\title{
Hybrid renewable energy system for ocean going platforms
}

\author{
$\mathrm{Yu} \mathrm{Cao}$ \\ Fluid Structure \\ Interactions Group \\ University of Southampton \\ Southampton, United Kingdom \\ Email: Yu.Cao@soton.ac.uk
}

\author{
Nicholas Townsend \\ Fluid Structure \\ Interactions Group \\ University of Southampton \\ Southampton, United Kingdom \\ Email: Nick@soton.ac.uk
}

\author{
Mingyi Tan \\ Fluid Structure \\ Interactions Group \\ University of Southampton \\ Southampton, United Kingdom \\ Email: M.Tan@soton.ac.uk
}

\begin{abstract}
Renewable energy systems that extract power from sustainable sources such as wind, wave and solar can provide clean energy to a range of marine applications. Currently a major challenge is in providing an uninterrupted power supply, given the variation and uncertainty in these renewable energy sources. To deal with the unpredictable nature of renewable energy resources, this paper investigates the use of hybrid systems (systems that harvest multiple sources) to reduce the variation and uncertainty of the power generated. Considering the use of multiple renewable energy resources including solar, wind and wave, this paper first evaluates the available energy resources and the technical recoverable power using open-source, yearly, global, meteorological datasets. Based on the technical recoverable potential the complimentary features between solar and wind resources are investigated and compared to the propulsive power demand of various sized ocean going platforms and routes. The analysis shows that the resources can be complementary and that hybrid systems are more suitable for small vehicles, craft or maritime robotics system such as autonomous surface and underwater vehicles and oceanographic buoys.
\end{abstract}

Index Terms-Hybrid renewable energy, maritime robotics, slow steaming, energy harvesting, energy scavenging

\section{INTRODUCTION}

Renewable energy systems that extract power from sustainable sources such as solar, wind and wave can supply power with low negative environmental impact [1] and promise energy efficient operation(s). To date various renewable energy systems have been developed for a range of marine applications, however, most studies are focused on the design of the energy conversion device for a single energy resource i.e., solar ( [2], [3]), wind ( [4], [5]) and wave ( [6]-[10]). With the variable nature of renewable energy resources, a major challenge for single resource systems is the intermittent power supply and increased capital cost of the power storage and management systems. To improve the energy supply stability, this paper considers the use of a hybrid system that combines multiple sources together.

In this study, global, multi-year, averaged, meteorological data has been used to estimate the environmental resource potential (the theoretical or upper limit of power that is available in the natural environment). Then incorporating renewable energy harvesting models, the technical (or realistic) recoverable power has been identified. Based on the technical recoverable energy between multiple renewable resources, the complimentary features between the different resource harvesting has been identified. The recoverable power is also compared to the propulsive load of various sized ocean going platforms and routes, identifying the potential of hybrid renewable energy systems.

The paper is structured as follow; Section II details the available resources and technical recoverable renewable energy and Section III presents the assessment of the complimentary features and comparison of the generated power to platform propulsive power demand.

\section{RENEWABLE ENERGY RESOURCES}

\section{A. Available Resources}

The available energy resource, (i.e., the theoretical or upper limit of power that is available in the natural environment), across the oceans are presented next based on global, multiyear, averaged, meteorological data.

1) Solar: The solar renewable energy resource, the short-wave radiation and sensible heat that is received on the surface of the earth, varies both temporally and spatially on the Earth's surface. A 22 year annual average Global Horizontal Irradiance (GHI), the total shortwave radiation received on a surface horizontal to the surface of the earth, from NASA Surface meteorology and Solar Energy database [11] is presented in 1. 1, the averaged solar energy density, shows that the average solar radiation level generally decreases from the equator towards the poles, with averaged solar radiation levels of approximately $60 \mathrm{~W} / \mathrm{m}^{2}$ near the North and South poles. Regions such as the Indian ocean, Southern Atlantic ocean, and parts of Pacific ocean have a high concentration of solar energy of over $280 \mathrm{~W} / \mathrm{m}^{2}$. Ocean solar radiation levels are also influenced by cloud-cover. For example, the small area in the tropical Western Pacific does not follow the general trend, due to cloud-cover [12].

Horizontal solar irradiance levels also follow seasonal and daily cycles, as shown in 2. A diurnal cycle of solar 


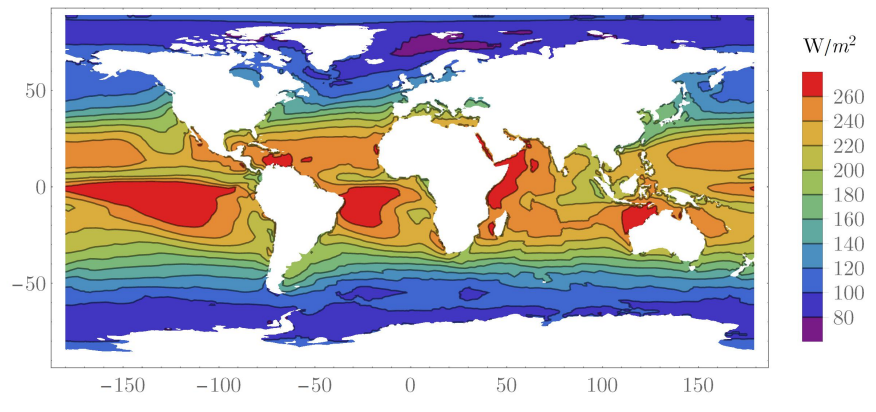

Fig. 1. Global horizontal irradiance. Data source: NASA SSE

radiation can be found during day and night: with a peak solar radiation level at noon time. A seasonal cycle results in the Northern Hemisphere having a higher radiation level in summer time (and vice versa, the South Hemisphere has a higher radiation level during winter).

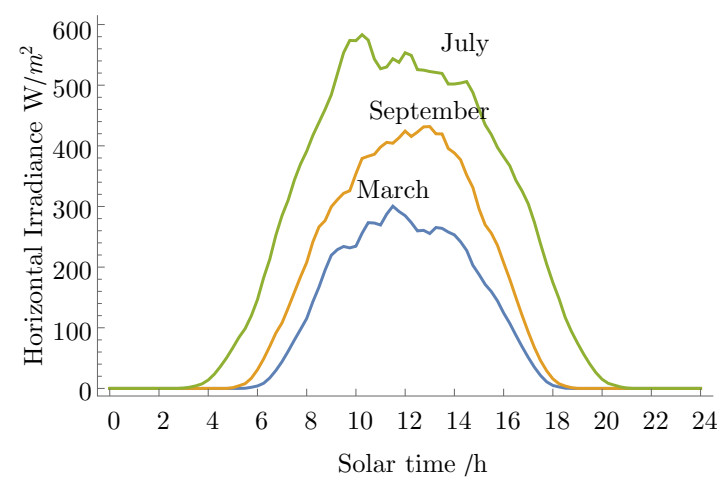

Fig. 2. Daily averaged solar radiation at typical month $\left(56.5^{\circ} \mathrm{N}, 3.25^{\circ} \mathrm{E}\right)$

2) Wind: Similar to solar, wind is also highly variable. Wind speed is characterized by high temporal variations, as shown in 3, and spatial variations, as shown in 4. Temporally, wind speed is influenced by micro-meteorological variations known as turbulence which range from a few seconds to a couple of minutes (as shown by the first peak between 0.01 to 0.02 hours in 3), and macro-meteorological affects (i.e., diurnal and annual patterns). Diurnal cycles are mainly caused by temperature differences between day and night and annual cycles are due to the relative motion between sun and earth.

As wind is mainly driven by atmospheric temperature differences, higher averaged wind speeds are typically found between air masses with larger temperature differences. The geographic variation of wind speed, shown in 4, varies largely with latitude, with a notable peak in the South Hemisphere.

As the wind energy is related to wind speed as:

$$
\mathcal{P}_{\text {wind }}=\frac{1}{2} \rho V^{3}
$$

where $\rho$ is the density of air, the variations in wind speed become more pronounced in terms of power.

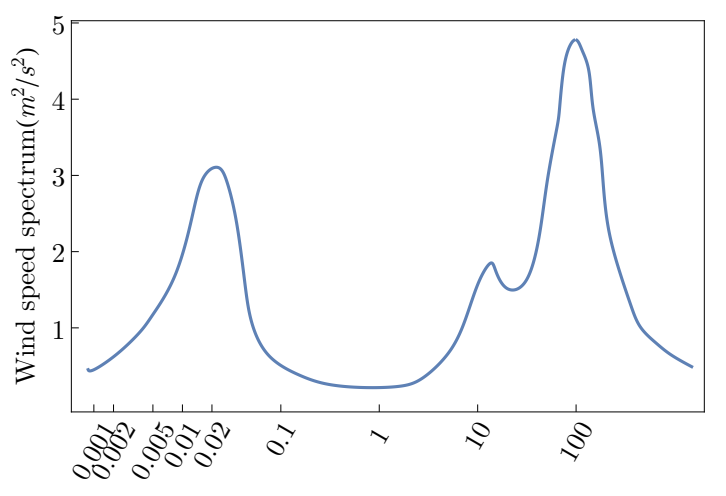

Hour(h)

Fig. 3. Horizontal wind speed spectrum example. Data source: Brookhaven National Laboratory

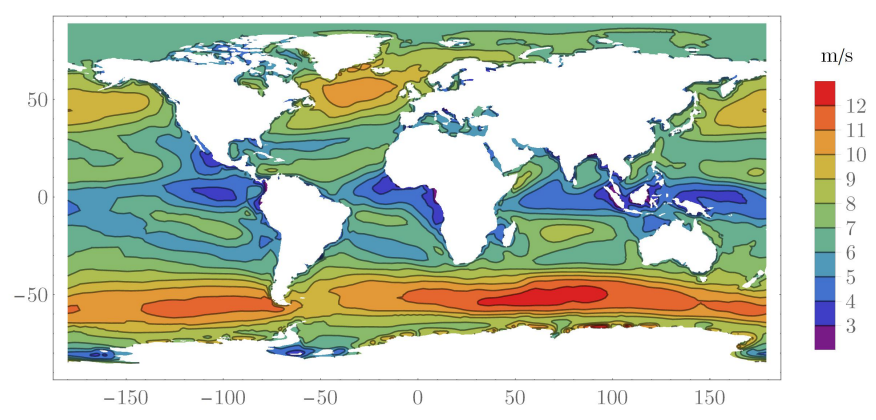

Fig. 4. Global wind speed distribution. Data source: NASA SSE

3) Wave: Waves, created by the wind passing over the surface of the sea and varying spatially and temporally, have the highest energy density among the renewable resources considered in this paper. It has been proven that in the case of fully developed wind sea with sufficiently long fetch [13], modeled with the semi-empirical Pierson-Moskowitz spectrum

$$
S(f)=\left(A / f^{5}\right) \exp \left(-B / f^{4}\right)
$$

The wave power flow intensity is maximum when

$$
I(0)=2 \pi \rho g m_{1}=\frac{\pi}{8} \rho g H_{m 0}^{2} / T
$$

where $m_{j}$ is defined as spectral moment

$$
m_{j}=\int_{0}^{\infty} f^{j} S(f) d f
$$

Where $H_{m 0}$ represents the significant wave height for zero order moment $m_{0}$. Considering, a fully developed sea and the energy flux just under the surface, then $H_{m 0}=4 \sqrt{m_{0}}$ and

$$
I(0)=0.0325 \rho g^{3 / 2} H_{m 0}^{3 / 2} \approx 5 I_{\text {wind }}
$$

That is, the distribution of wave and wind energy are similar, as can be seen by comparing the global wind speed distribution (4) and global annual mean wave power density (5). As a result, and partly because of the limited availability of opensource monthly wave spectrum data, a full analysis of wave 
energy has been omitted, as it can be correlated to the wind energy potential.

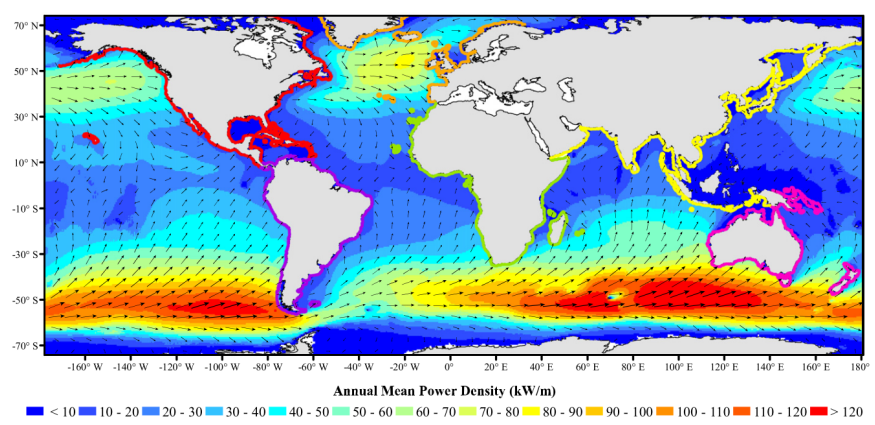

Fig. 5. Global annual mean wave power density [14]

\section{B. Recoverable Power}

To assess the potential of hybrid energy harvesting for ocean going platforms, two characteristics are important: 1) the total amount of energy that can be (practically) recovered from the renewable resource, and 2) the variation (spatial and temporal) in the recovered power (that impacts the power supply consistency). The recoverable solar and wind resources are considered next.

1) Solar: Modeling a solar panel as an ideal electric generator with optimal tracking control:

$$
P(G)=\eta_{s} \cdot A \cdot G
$$

where $A$ is area of solar panel, $G$ is global horizontal irradiance and $\eta_{s}$ is the energy conversion efficiency (determined by the type and working conditions of solar panel). Then the global solar power density, assuming a constant energy conversion efficiency of $16 \%$ (considered as an average, current commercially available system), is that as presented in 6 . The recoverable power varies primarily with latitude, ranging from 10 to $50 \mathrm{~W} / \mathrm{m}^{2}$ approximately.

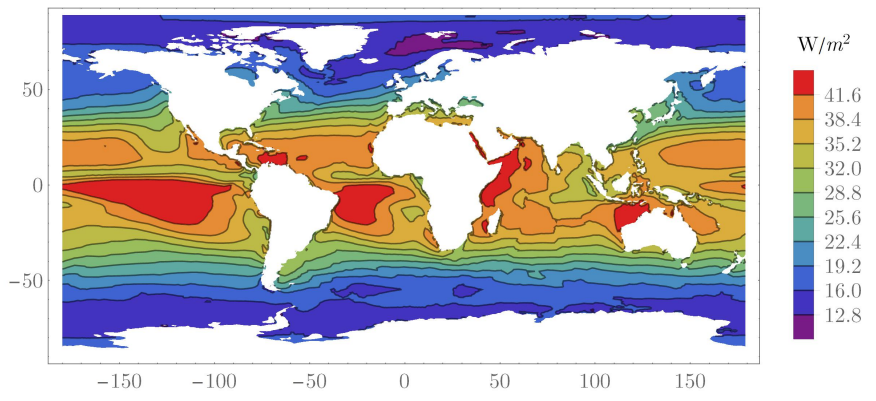

Fig. 6. Estimated global recoverable solar power

2) Wind: Assuming an ideal wind turbine model with cutin and cut-off control constructed as a piecewise function to calculate wind power generation:

$$
P(v)= \begin{cases}0 & v \leq v_{\text {cut-in }} \\ \frac{1}{2} \rho \cdot C_{p}(\lambda) \cdot A \cdot v^{3} & v_{\text {cut-in }} \leq v \leq v_{\text {rated }} \\ \frac{1}{2} \rho \cdot C_{p}(\lambda) \cdot A \cdot v_{\text {rated }}^{3} & v_{\text {rated }} \leq v \leq v_{\text {cut-off }} \\ 0 & v>v_{\text {cut-off }}\end{cases}
$$

where $v$ is wind speed, $\rho$ is the density of air, $A$ is swept area of wind turbine and $C_{p}(\lambda)$ is the power coefficient of the wind turbine where $\lambda$ is tip speed ratio $v / \omega R$ of wind turbine (that can either be actively controlled or passively driven based on the design of turbine system).

Considering a common three blade horizontal axis wind turbine and assuming a typical averaged $C_{p}$ value of 0.26 (i.e., an active speed controlled system to maintain performance) and cut-off model. The recoverable wind power can be estimated, as that shown in 7 . The maximum recoverable wind power, of $230 \mathrm{~W} / \mathrm{m}^{2}$, is found in the southern hemisphere. In the northern hemisphere the wind power ranges from 20 to $150 \mathrm{~W} / \mathrm{m}^{2}$ approximately varying with latitude.

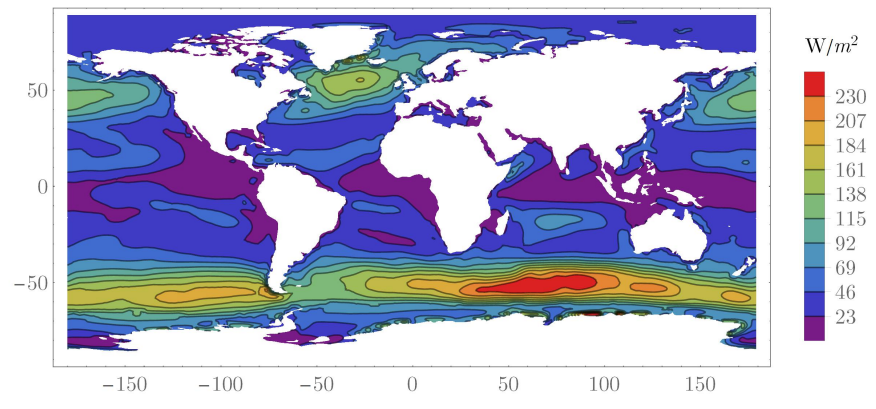

Fig. 7. Estimated global recoverable wind power

\section{HYBRID SYSTEM ASSESSMENT}

\section{A. Complementarity}

The principle of a hybrid system is to harvest complimentary, multiple resources, to improve the energy supply stability (and hopefully reduce buffer requirements and power management complexity). For example, as shown in $8\left(55^{\circ} \mathrm{E}, 10^{\circ} \mathrm{N}\right.$ at North Hemisphere) solar energy has a lower energy density during winter. Wind energy could compensate such temporal variation during the winter since it has a higher energy density during winter.

To find the global complimentary features of solar and wind renewable (recoverable) energy resources, a statistical correlation was made using the Kendall Tau rank correlation. (Note: as the distribution of wave and wind is similar, this study of wind-solar complementarity should also apply to a wave-solar resource mix). The Kendall Tau rank correlation provides a correlation index between two variables, equal to 1 for similar rank and -1 for a fully different rank. 
TABLE I

KENDALL TAU TABLE AT SAMPLE LOCATION

\begin{tabular}{l|ccccccccccccc}
\hline Month & 1 & 2 & 3 & 4 & 5 & 6 & 7 & 8 & 9 & 10 & 11 & 12 & \\
\hline Solar & 4.13 & 8.80 & 16.6 & 26.4 & 35.7 & 37.1 & 35.9 & 29.9 & 19.4 & 10.5 & 5.27 & 3.33 & \\
Wind & 40.3 & 24.1 & 23.0 & 15.9 & 12.7 & 11.1 & 13.9 & 13.3 & 18.4 & 23.1 & 23.6 & 28.6 & Total \\
\hline Concordant & 1 & 1 & 0 & 0 & 1 & 0 & 1 & 0 & 0 & 0 & 0 & 0 & 4 \\
Discordant & 10 & 9 & 9 & 8 & 6 & 6 & 4 & 4 & 3 & 2 & 1 & 0 & 62 \\
\hline
\end{tabular}

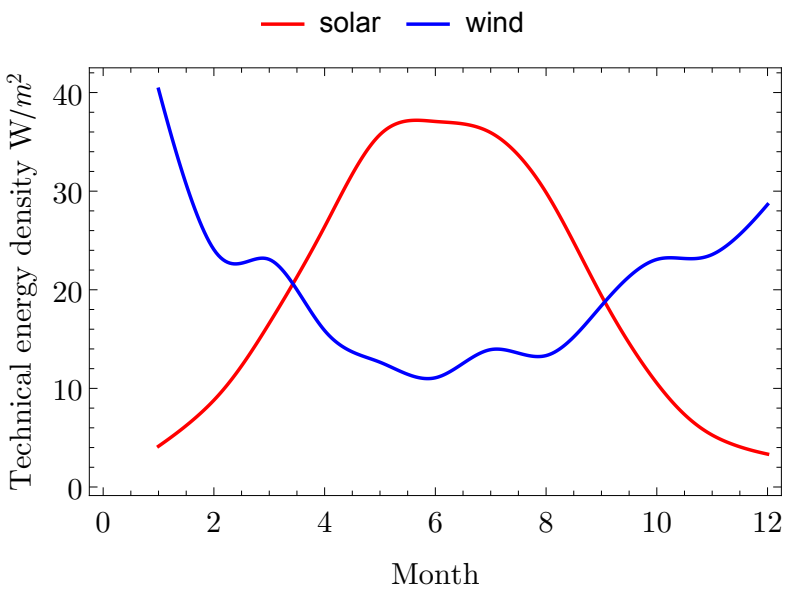

Fig. 8. Sample multi-year monthly average technical density at $\left(55^{\circ} \mathrm{E}, 10^{\circ} \mathrm{N}\right)$

An example of Kendall $\tau$ correlation is shown in I. Here $s_{i}$ and $w_{i}$ are the solar and wind recoverable power densities, for month $i$. Concordant pairs for $\left(s_{i}, w_{i}\right)$ are the number of pairs that $\left(s_{j}, s_{k}\right)$ and $\left(w_{j}, w_{k}\right)$ are of the same rank. Vice versa, discordant pairs are the number of pairs that $\left(s_{j}, s_{k}\right)$ and $\left(w_{j}, w_{k}\right)$ are of different rank. For example, $\left(s_{1}, w_{1}\right)$ has 1 concordant pair which is $\left(s_{1}, s_{12}\right)$ and $\left(w_{1}, w_{12}\right)$ because $s_{1}>$ $s_{12}$ and $w_{1}>w_{12}$. The other 10 pairs are discordant because they have different rank. Then by adding up the numbers of concordant pairs $C$, and discordant pairs $D$, Kendall $\tau$ can then be defined and calculated as;

$$
\tau=\frac{C-D}{C+D}
$$

Kendall $\tau$, a number between -1 to 1 , shows how similar or different the two datasets are. In this case, a negative value means two renewable resources are likely to be complimentary to each other. A positive number indicates a similar trend of the two renewable energy resources. For example, the Kendall $\tau$ number for the example presented in 8 is -0.88 , showing a complimentary feature in resources, as expected.

1) Global Kendall $\tau$ : Using this method, a global correlation test was performed based on $1^{\circ}$ by $1^{\circ}$ ocean regions and the multi-year, monthly average recoverable power densities for solar and wind. The histogram of global Kendall $\tau$ values (9) shows that the majority of the oceans have a temporal complimentary feature, with an average (mode) Kendall $\tau$ value of -0.7 .

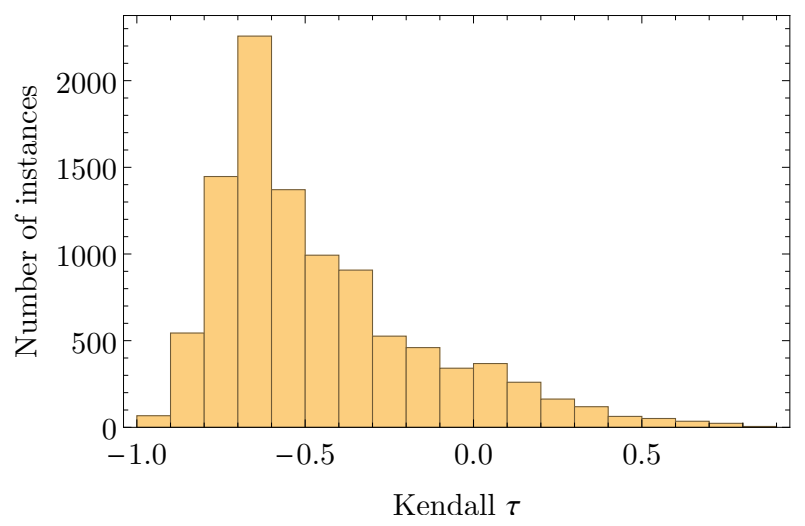

Fig. 9. Histogram of global Kendall $\tau$ of wind and solar power

2) Geographical distribution of Kendall $\tau$ : The geographical distribution of Kendall $\tau$ is shown in 10 . The regions with negative Kendall $\tau$ imply that wind-solar hybrid renewable energy system for ocean going platforms are suitable. That is, the wind and solar power is more likely to compensate each other and provide a smoother (less variable) power supply for the platform. 10 also shows that the majority of the oceans are complimentary in solar and wind power, most notably in the South Pacific ocean, India ocean and North Atlantic ocean. Although, it should be noted that it does not necessary mean regions with positive Kendall $\tau$ are not suitable for a wind-solar hybrid system. Rather, this indicates in those regions, the individual resources within the hybrid system are unlikely to be able to compensate (complement) each other on a monthly time scale.

3) Monthly Distribution of Kendall $\tau$ : A monthly distribution of Kendall $\tau$ test was also calculated, comparing the annual solar and wind recoverable power densities. 11 shows that the Kendall $\tau$ varies with month and that the averaged value is below zero. These complimentary features suggest the use of a hybrid renewable energy system. However, most ocean going platforms operate on specific route(s) or region(s), so ideally a more refined Kendall $\tau$ index may be required/useful.

\section{B. Propulsion and Recoverable Power Comparison}

1) Propulsion Power: The propulsion load of ocean going platforms was estimated, for various platform lengths, 


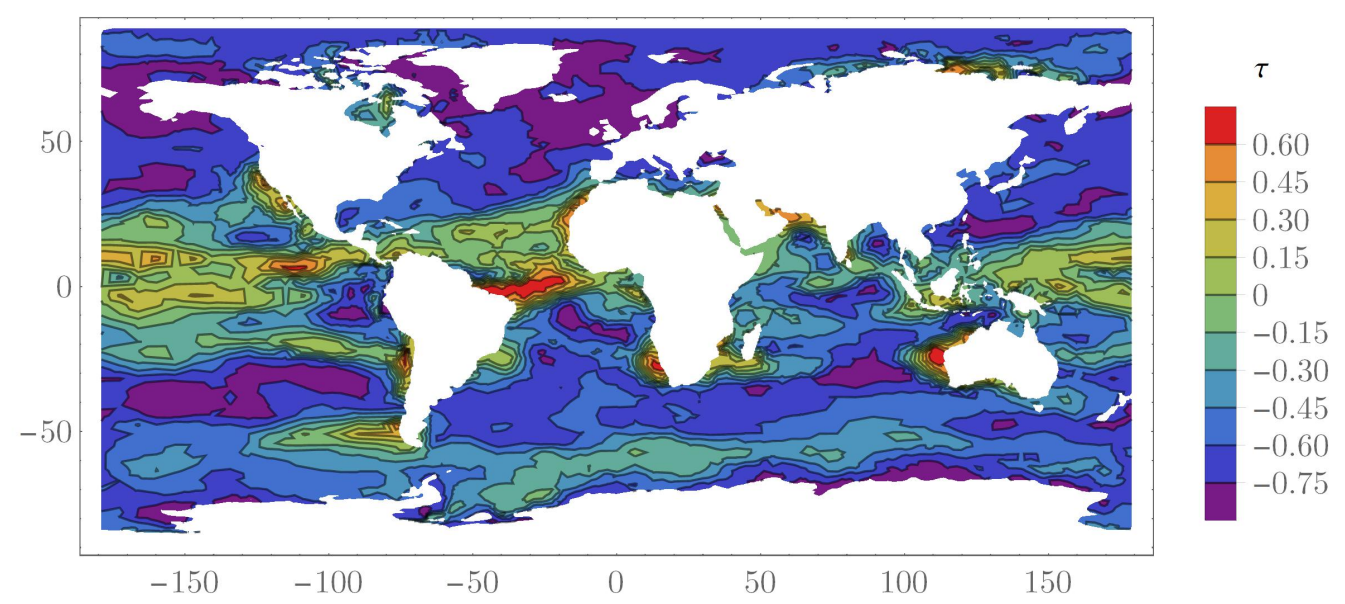

Fig. 10. Global Kendall $\tau$ of wind and solar power

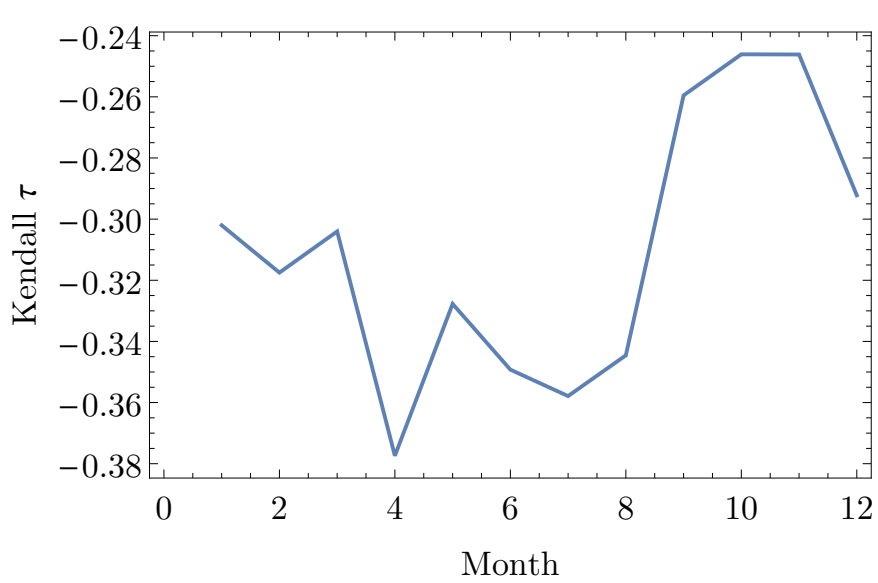

Fig. 11. Monthly global Kendall $\tau$

assuming ship-shape platforms (i.e., a slender body moving in one direction) using the Harvald regression method present by Kristensen [15]. This method, as shown in 12, divides the propulsion resistance, equation, into four groups: frictional resistance, residual resistance, added roughness resistance and air resistance.

Assuming a $20 \%$ margin to take account additional wave load on ship, the total resistance power of a 'platform' (at a certain length) was determined as:

$$
P_{\text {prop }}=(1+20 \%) \cdot \frac{1}{2} \rho S V^{2}\left(C_{F}+C_{R}+C_{A}+C_{A A}\right)
$$

$S$ the surface area was modelled using Mumford's formula:

$$
S=1.025 \cdot\left(\frac{\Delta}{T}+1.7 \cdot L_{p p} \cdot T\right)
$$

$C_{F}$ the frictional resistance coefficient that indicates the sum of tangential stresses along the wet surface along the direction of motion, was modelled according to ITTC-57:

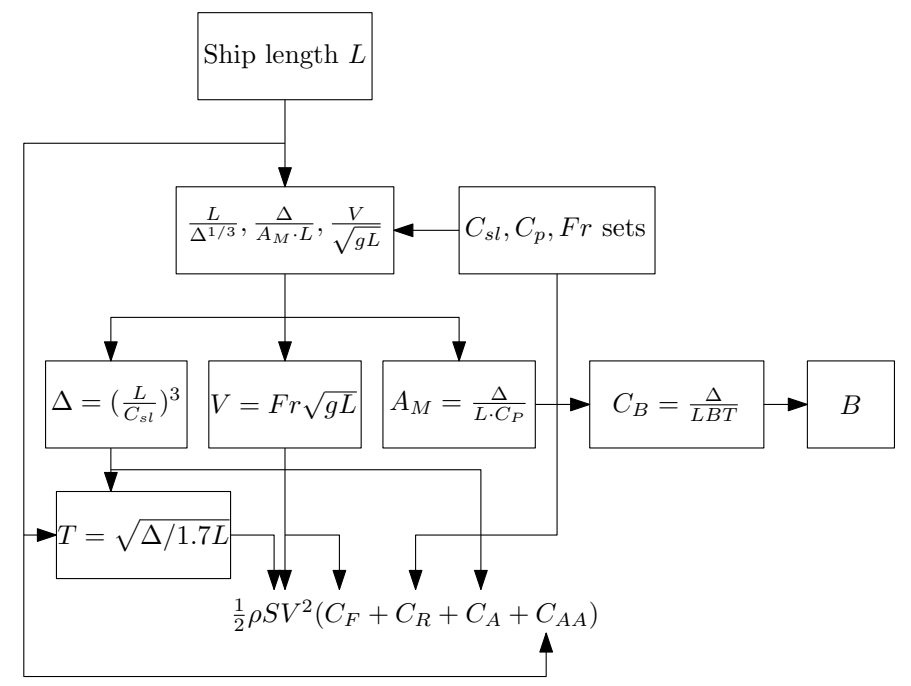

Fig. 12. Flow diagram of estimating propulsion load for a length with all possible $C_{R}$

$$
C_{F}=\frac{0.075}{\left(\log R_{n}-2\right)^{3}}
$$

Where $R_{n}$ is the Reynolds number that correlates the ship speed $V$, length $L$ and viscosity $\nu$ of sea water: $R_{n}=\frac{V \cdot L}{\nu}$.

$C_{R}$ is the residual resistance coefficient, the combination of pressure force normal to the surface of the hull, was determined using the empirical Harvald method. This method estimates the ship propulsion with geometry parameters including slenderness function $C_{s l}=L / \Delta^{1 / 3}$, prismatic coefficient $C_{P}=\Delta / A_{M} \cdot L$ and operating parameters including Froude number $\mathrm{Fr}=V / \sqrt{g L}$. For all parameters within the domain of $C_{s l}, C_{P}, \operatorname{Fr}$ the residual resistance coefficient $C_{R}\left(C_{s l}, C_{P}, \mathrm{Fr}\right)$ were found using a linear interpolation lookup table method. $C_{A A}$ is air added resistance due to the drag of ship superstructure. 
2) Recoverable Power: Assuming a simplified linear harvesting model, then the total recoverable power that can be harvested is:

$$
P=\delta A
$$

where $\delta$ is technical recoverable energy density unit in $W / m^{2}$ and $A$ represents the available space (geometry) for the harvesters . To scale the recoverable power to a specific platform size, the following assumptions were made.

For solar energy, the maximum area for energy harvesting on board was assumed not to exceed the deck area, given by;

$$
A_{\text {solar }} \leq C_{W} \cdot L B
$$

where $C_{W}$ is water plane area coefficient, the ratio of area of water-plane to the product of ship length and beam.

For wind energy, considering the spacing and interactions between wind turbines, the total number of turbines was considered as $\min (3, L / B)$, with each turbine the swept area as wide as $70 \%$ beam $B$ and as tall as twice of draft $T$. That is;

$$
A_{\text {wind }} \leq \min (3, \underline{L / B}) \cdot 0.7 B \cdot 2 T
$$

here $L / B$ represents the floor function to ensure the number of turbines is an integer.

3) Comparison: Given the 'platform' geometry parameters (the block coefficient, $C_{B}$ ) the total recoverable power can be estimated as;

$$
P_{\text {supply }} \leq \delta_{\text {solar }} C_{W} \cdot L B+\delta_{\text {wind }} \cdot \min (3, \underline{L / B}) \cdot 0.7 B \cdot 2 T
$$

and the ratio of recoverable to propulsion power can then be estimated as:

$$
\frac{P_{\text {supply }}}{P_{\text {load }}}=\frac{\delta_{\text {solar }} C_{W} \cdot L B+\delta_{\text {wind }} \cdot \min (3, L / B) \cdot 0.7 B \cdot 2 T}{1.2 \cdot \frac{1}{2} \rho S V^{2}\left(C_{F}+C_{R}+C_{A}+C_{A A}\right)}
$$

Assuming a block coefficient of 0.8 , and considering three example routes (Europe to North America, Middle East to North America, Far East to America), 13. The estimated renewable energy harvesting potential, for a range of platform lengths is that presented in 14. (The slenderness coefficient, prismatic coefficient and Froude number are in the domain of 4 to $8,0.5$ to 0.8 and 0.15 to 0.45 correspondingly).

\section{Discussion}

The estimated recoverable renewable power from multiple sources shows that there is generally a complimentary trend between solar-wind power, and solar-wave power (assuming a fully developed sea). While, wind and wave energy was found to follow a similar monthly trend, with no complementarity features. This suggests that a hybrid system of wind-solar or

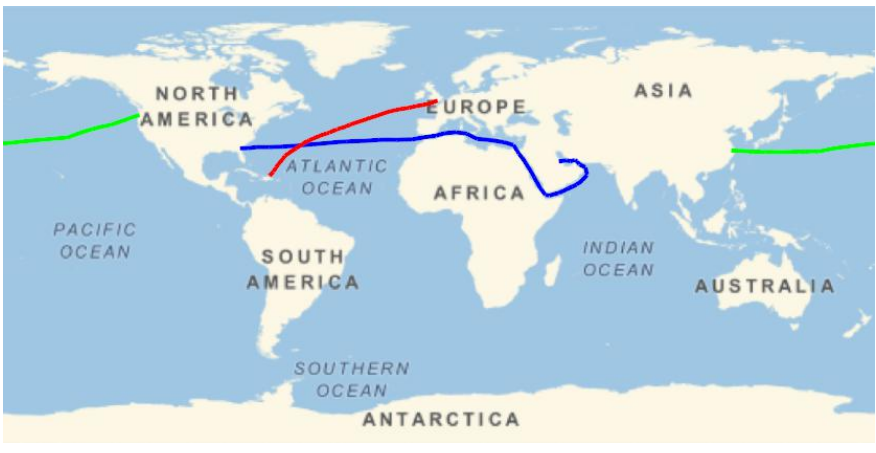

Fig. 13. Three typical routes for study

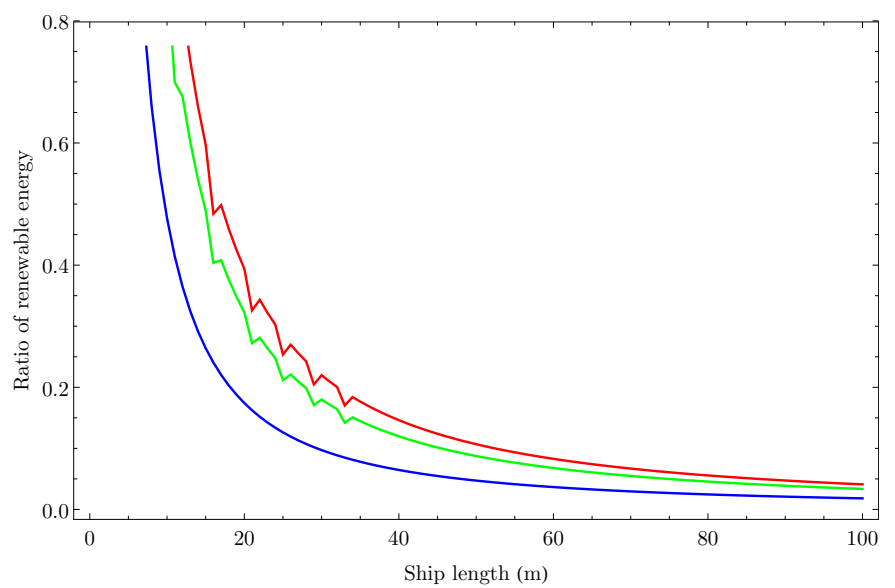

Fig. 14. Fraction between renewable power of propulsion power for three (red, green, blue) routes

wave-solar, could provide a more robust power supply, with smaller energy storage requirements.

Comparing the renewable, recoverable power to the estimated propulsive power requirements for a range of platform sizes, the results show that a hybrid system can provide a significant proportion of the propulsion power at smaller scales (e.g., under $10 \mathrm{~m}$ ). For a platform of around 100 meters, the contribution of renewable power (compared to propulsive power) is only around 1 to 3 percent. Therefore, for larger commercial vessels operating at their service speed, the total renewable energy compared to the propulsion demand is low and a hybrid system may be more suitable to augment the hotel load. The results also suggest a hybrid renewable design could lead to a small ocean going platform of 1 to 4 meters that can achieve fully autonomous, self-maintained operation.

\section{CONCLUSION}

This paper investigated the use of hybrid systems (systems that harvest multiple sources) to reduce the variation and uncertainty of the renewable power generated for ocean going platforms. In general, wind and solar were found to be complimentary and that a hybrid wind-solar energy harvesting system 
can smooth out the monthly variation of these renewable resources. The results show that hybrid systems, given temporal and spatial variations, can improve the energy supply stability, and may increase the average power supply level and maintain a relative constant (averaged) power supply from renewable resources for a specific route or operating region. Compared to the propulsive power, the results also show that hybrid systems are likely to be more suitable for smaller vehicles such as autonomous surface vehicles and oceanographic buoys.

\section{ACKNOWLEDGMENT}

This research was supported by the Lloyds Register Foundation (LRF).

\section{REFERENCES}

[1] Winebrake, James J., James J. Corbett, and Patrick E. Meyer. "Energy use and emissions from marine vessels: a total fuel life cycle approach." Journal of the Air \& Waste Management Association 57.1: 102-110, 2007.

[2] Crimmins, Denise M., et al. "Long-endurance test results of the solarpowered AUV system." OCEANS' 2006. IEEE, 2006.

[3] Spagnolo, Giuseppe Schirripa, et al. "Solar-Electric Boat." Journal of Transportation Technologies 2.02: 144, 2012.

[4] Dadd, George M., Dominic A. Hudson, and R. A. Shenoi. "Determination of kite forces using three-dimensional flight trajectories for ship propulsion." Renewable Energy 36.10: 2667-2678, 2011.

[5] Traut, Michael, et al. "Propulsive power contribution of a kite and a Flettner rotor on selected shipping routes." Applied Energy 113: 362372,2014

[6] Manley, Justin, and Scott Willcox. "The wave glider: A persistent platform for ocean science." OCEANS' 2010 IEEE-Sydney. IEEE, 2010.

[7] Bowker, J.A., Townsend, N.C., Tan, M. and Shenoi, R.A. "Experimental analysis of submerged flapping foils; implications for autonomous surface vehicles (ASVs)" OCEANS'16 MTS/IEEE, IEEE, 2016.

[8] Townsend, Nicholas. "Self powered Autonomous Underwater Vehicles (AUVs): Results from a gyroscopic energy scavenging prototype" IET Renewable Power Generation, 10(8):1078, 2016.

[9] Hagerman, George. "Wave energy systems for recharging AUV energy supplies." In Proceedings of the 2002 Workshop on Autonomous Underwater Vehicles, IEEE, 2002.

[10] Tiao, Wen-Chuan. "Preliminary assessments of the anti-roll tank as a wave energy converter." Ships and Offshore Structures 8.5: 488-496, 2013.

[11] NASA LaRC POWER Project. "Surface meteorology and Solar Energy database." [Online] Available: https://eosweb.larc.nasa.gov/cgi-bin/sse/ sse.cgi [Accessed: 6-Apr-2017] , 2015.

[12] Webster, Peter J., Carol Anne Clayson, and Judith A. Curry. "Clouds, radiation, and the diurnal cycle of sea surface temperature in the tropical western Pacific." Journal of Climate 9.8: 1712-1730, 1996.

[13] Johannes Falnes. "A review of wave-energy extraction." Marine Structures, 20.4: 185-201, 2007.

[14] Kester Gunn, Clym Stock-Williams. "Quantifying the global wave power resourc.," Renewable Energy, 44.C: 296-304, 2012.

[15] Kristensen, Hans Otto, and Marie Ltzen."Prediction of Resistance and Propulsion Power of Ships." Clean Shipping Currents , 1.6: 2012. 